\title{
Twenty-years' use of expanded polytetrafluoroethylene sheet for an artificial cardiac pacemaker
}

\author{
Kenji Yorita ${ }^{1}$, Junichi Takahashi ${ }^{1}$, Kazutoshi Tano ${ }^{1}$, Yoichi Ichikawa ${ }^{1}$, Nobumasa \\ Hamaguchi $^{1}$, and Wataru Yasui ${ }^{2}$ \\ ${ }^{1}$ Japanese Red Cross Kochi Hospital \\ ${ }^{2}$ Yusuhara Hospital
}

March 6, 2021

\section{Clinical Images}

Title: Twenty-years' use of expanded polytetrafluoroethylene sheet for an artificial cardiac pacemaker

Kenji Yorita ${ }^{1 *}$, Junichi Takahashi ${ }^{2}$, Kazutoshi Tano ${ }^{3}$, Yoichi Ichikawa ${ }^{3}$, Nobumasa Hamaguchi ${ }^{4}$, Wataru Yasui $^{5}$

${ }^{1}$ Department of Diagnostic Pathology, Japanese Red Cross Kochi Hospital, Kochi-city, Kochi, Japan

${ }^{2}$ Department of Internal Medicine, Japanese Red Cross Kochi Hospital, Kochi-city, Kochi, Japan

${ }^{3}$ Department of Cardiovascular Surgery, Japanese Red Cross Kochi Hospital, Kochi-city, Kochi, Japan

${ }^{4}$ Department of Thoracic Surgery, Japanese Red Cross Kochi Hospital, Kochi-city, Kochi, Japan

${ }^{5}$ Department of Internal Medicine, Yusuhara Hospital, Kochi-city, Kochi, Japan

The authors report no conflict of interest, financial or otherwise.

${ }^{*}$ Corresponding Author: Kenji Yorita, M.D., Ph.D.,

Department of Diagnostic Pathology, Japanese Red Cross Kochi Hospital, 1-4-63-11, Hadaminamimachi, Kochi-city, Kochi 780-8562, Japan

Tel: $+81-88-822-1201$

Fax: $+81-88-822-1056$

E-mail: kenjiyorita@gmail.com

Keywords : expanded polytetrafluoroethylene sheet, cardiac pacemaker, foreign body

\section{Key Clinical Message}

Physicians can prolongedly use expanded polytetrafluoroethylene sheets for fixation of artificial cardiac pacemakers to avoid pacemaker lead displacement. The sheets also can be used to prevent implant rejection in patients with metal allergies.

What material encases an artificial cardiac pacemaker?

An 84-year-old Japanese woman was admitted to our hospital to have her artificial cardiac pacemaker's battery replaced. The pacemaker had been inserted for sick sinus syndrome 20 years previously, and the 
battery had been replaced 11 years ago. The patient had a history of four surgeries for the adjustment of pacemaker lead position. During the second battery replacement, a physician noticed a hard, membranous material encasing the pacemaker. This material was removed (Figure 1A) to reduce the risk of infection. Histological analysis revealed a foreign body, which resembled an expanded polytetrafluoroethylene (ePTFE) sheet with a $0.1 \mathrm{~mm}$-thick laminar structure, embedded in a fibrous stroma (Figure $1 \mathrm{~B}-1 \mathrm{C}) .{ }^{1} \mathrm{Although}$ the data concerning the insertion of ePTFE is now unavailable, it is known that ePTFE had been probably used for 20 years. The purpose of the use is unknown; however, ePTFE sheets were likely used for fixation of the pacemaker to avoid pacemaker lead displacement. The sheets also can be used to prevent implant rejection in patients with metal allergies. ${ }^{2}$ Our case likely demonstrates the safety of prolonged use of ePTFE sheets with artificial pacemakers.

\section{AUTHOR CONTRIBUTIONS}

KY: drafted the manuscript and contributed to the pathological diagnosis of the foreign body. JT: treated the patient and removed the foreign body. KT, YI, and NH: assisted with the analysis and interpretation of the foreign body. WY: acquired the data and obtained informed written consent from the patient for the publication of the clinical image.

\section{CONFLICT OF INTEREST}

The authors state that they have no conflicts of interest to declare or funding resources to mention.

ORCID ID

Kenji Yorita ID https://orcid. Org/0000-0001-6428-0222

\section{Acknowledgements}

We would like to thank Ms. Keiko Mizuno, Mr. Masahiko Ohara, Ms. Kaori Yasuoka, Ms. Yukari Wada, and Mr. Hiroyuki Tsutsui for preparing the histological specimens.

\section{References}

1 Naito Y, Shin'oka T, Hibino N, Matsumura G, Kurosawa H. 2008. A novel method to reduce pericardial adhesion: a combination technique with hyaluronic acid biocompatible membrane. J Thorac Cardiovasc Surg. 135:850-856.

2. Ishii K, Kodani E, Miyamoto S, et al. 2006. Pacemaker contact dermatitis: The effective use of a polytetrafluoroethylene sheet. Pacing Clin Electrophysiol. 29:1299-1302.

\section{FIGURE LEGENDS}

Figure 1

A removed membranous material, which encased the cardiac pacemaker is seen (A ). Histologically, a foreign body with a thickness of $0.1 \mathrm{~mm}$ is embedded in a dense fibrous stroma (B ). The material and calcification are indicated by arrows and arrowheads, respectively. The higher magnification image of the material shows a laminar structure indicated by asterisks. Scale bar in A, B, and C is $1 \mathrm{~cm}, 0.1 \mathrm{~mm}$, and $0.1 \mathrm{~mm}$, respectively. 

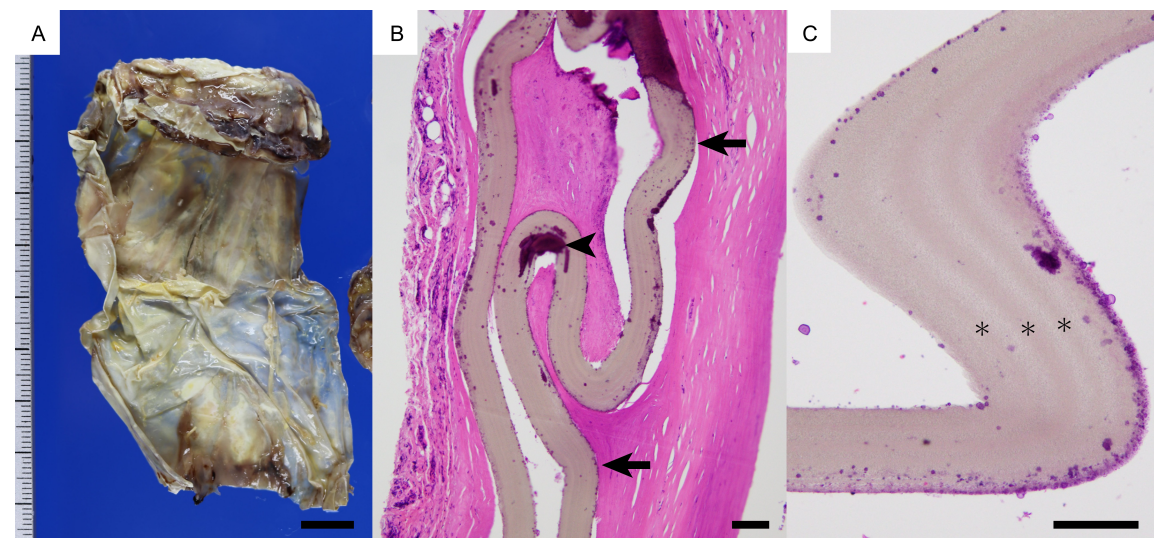